\title{
Magnetically assisted explosions of weakly magnetized stars
}

\author{
Hidetomo Sawai ${ }^{1,2}$ and Shoichi Yamada ${ }^{2}$ \\ ${ }^{1}$ Research Organization for Information Science \& Technology, \\ Minatojima-Minamimachi 1-5-2, Chuo, Kobe, Hyogo, Japan \\ email: hsawai@rist.or.jp \\ ${ }^{2}$ Scinence \& Engineering, Waseda University, \\ Okubo 3-4-1, Shinjuku, Tokyo, Japan
}

\begin{abstract}
We carried out high resolution simulations of weakly-magnetized core-collapse supernovae in two-dimensional axisymmetry in order to see the influence of the magnetic field and rotation on the explosion. We found that the magnetic field amplified by magnetorotational instability (MRI) has a great positive impact on the explosion by enhancing the neutrino heating, provided that the progenitor has large angular momentum close to the highest value found in stellar evolution calculations. We also found that even for progenitors neither involving strong magnetic flux nor large angular momentum, the magnetic field is greatly amplified by the convection aand rotation, and this leads to the boost of the explosion again by enhancing the neutrino heating.
\end{abstract}

Keywords. supernovae: general, MHD, instabilities, methods: numerical

\section{Introduction}

The explosion mechanism of core-collapse supernovae has not yet been fully understood. Although the neutrino heating is likely the most promising mechanism, state-ofthe-art simulations led to less energetic explosions than what are observed in reality, and in some cases even resulted in no explosion (e.g., the article by E. O'Connor in this proceedings).

The impacts of magnetic field and rotation on core-collapse supernovae have been studied as possible agents to drive explosion other than neutrino heating. Previous studies showed that an energetic explosion occurs when a pre-collapse iron core involves a strong magnetic field, $B$ pre $\sim 10^{12}-10^{13} \mathrm{G}$, and rapid rotation, $\Omega$ pre $\sim 1 \mathrm{rad} \mathrm{s}^{-1}$ (e.g., Yamada \& Sawai 2004).

The strengths of the magnetic fields of pre-collapse cores are highly uncertain. However, it is likely that the above value of $B_{\text {pre }}$ is close to the extreme limit, since it corresponds to the magnetic flux comparable to those of magnetar candidates, the most highly magnetized stars ever known.

The rotation of $\Omega$ pre $\sim 1 \mathrm{rad} \mathrm{s}^{-1}$ may also be near the possible highest value. In stellar evolution calculations of Woosley \& Heger(2006), such a high speed rotation is obtained for main sequence stars with the surface rotational velocity more than a few $100 \mathrm{~km} \mathrm{~s}^{-1}$. Among the 216 O-type stars in 30 Dor analyzed by Ramírez-Agudelo et al. (2013) only the $25 \%$ of the sample have the projected surface velocity exceeding $200 \mathrm{~km} \mathrm{~s}^{-1}$.

In this article, we will see the dynamics of "weakly" magnetized core-collapse explosions based on magnetohydrodynamic (MHD) simulations, where $B_{\text {pre }}$ is one order of magnitude weaker than the above value. Numerical results are shown and discussed 
separately for fast-rotating progenitors (based on Sawai \& Yamada 2014, 2016) and for slowly-rotating progenitors (based on a work we are currently undertaking).

\section{Numerical Method}

We carry out two-dimensional simulations to follow the dynamics of $15 M_{\odot}$ star (Woosley \& Weaver 1995) for the central part within the radius of 500, 1000, or $4000 \mathrm{~km}$ covered with the polar coordinates by using a time-explicit Eulerian MHD code, Yamazakura (Sawai et al. 2013a). There the following MHD equations are solved with the assumptions of axisymmetry (and equatorial symmetry for the fast rotation case):

$$
\begin{aligned}
& \frac{\partial \rho}{\partial t}+\nabla \cdot(\rho \boldsymbol{v})=0, \\
& \frac{\partial}{\partial t}(\rho \boldsymbol{v})+\nabla \cdot\left(\rho \boldsymbol{v} \boldsymbol{v}-\frac{\boldsymbol{B} \boldsymbol{B}}{4 \pi}\right) \\
& \quad=-\nabla\left(p+\frac{B^{2}}{8 \pi}\right)-\rho \nabla \Phi, \\
& \frac{\partial}{\partial t}\left(e+\frac{\rho v^{2}}{2}+\frac{B^{2}}{8 \pi}\right) \\
& \quad+\nabla \cdot\left[\left(e+p+\frac{\rho v^{2}}{2}+\frac{B^{2}}{4 \pi}\right) \boldsymbol{v}-\frac{(\boldsymbol{v} \cdot \boldsymbol{B}) \boldsymbol{B}}{4 \pi}\right] \\
& \quad=-\rho(\nabla \Phi) \cdot \boldsymbol{v}+Q_{E}^{\mathrm{abs}}+Q_{E}^{\mathrm{em}}, \\
& \frac{\partial \boldsymbol{B}}{\partial t}=\nabla \times(\boldsymbol{v} \times \boldsymbol{B}), \\
& \frac{\partial n_{e}}{\partial t}+\nabla \cdot\left(n_{e} \boldsymbol{v}\right)=Q_{N}^{\mathrm{abs}}+Q_{N}^{\mathrm{em}},
\end{aligned}
$$

Here, $Q_{E}^{\mathrm{abs}}$ and $Q_{E}^{\mathrm{em}}$ are, respectively, the changes of energy density due to neutrino/antineutrino absorptions and emissions, which are given by simplified formulae of neutrino emission and absorption (Janka 2001). $Q_{N}^{\mathrm{abs}}$ and $Q_{N}^{\mathrm{em}}$ are the similar notations for the changes of electron number density. The electron fraction is required for the evaluations of these $\mathrm{Q}$ valuables, which is obtained by Eq. (2.5) after bounce, and by the prescription suggested by Liebendörfer(2005) before bounce. The gravitational potential, $\Phi$, is obtained by the Newtonian mono-pole formula. A tabulated nuclear equation of state produced by Shen et al. 1998a,b is utilized.

We assume that the pre-collapse core is rotating with the initial angular velocity profile of

$$
\Omega(r)=\Omega \text { pre } \frac{r_{0}^{2}}{r_{0}^{2}+r^{2}},
$$

where $r$ is the distance from the center of the core and $r_{0}=1000 \mathrm{~km}$. The angular velocity is set as $\Omega_{\text {pre }}=2.73$ and $0.1 \pi \mathrm{rad} \mathrm{s}^{-1}$ for rapid and slow rotation cases, respectively.

The pre-collapse magnetic field is assumed to be a dipole-like configuration produced by electric currents of a 2D-Gaussian-like distribution (see Sawai \& Yamada 2016 for the detailed formula). The strength of magnetic field at the center of the core is in the range of $5 \times 10^{10}-2 \times 10^{11} \mathrm{G}$.

We first run low resolution simulations for the numerical domain of $r<4000 \mathrm{~km}$ from the onset of the collapse to several $100 \mathrm{~ms}$ after bounce. The core is covered with 
$N_{r} \times N_{\theta}=720 \times 60$ (120 for full-sphere simulations) numerical grids, where the spatial resolution is $400 \mathrm{~m}$ near the center. Then, using the data obtained by the low resolution simulations as the initial and boundary conditions, we perform high resolution simulations starting from a few millisecond after bounce with smaller numerical domains $r_{\text {in }}<r<r_{\text {out }}$, where $\left(r_{\text {in }} / \mathrm{km}, r_{\text {out }} / \mathrm{km}\right)$ are $(50,500)$ and $(30,1000)$ for fast rotation simulations and slow rotation simulations, respectively. The number and the minimum size of numerical grids in the simulation of the highest numerical cost are, respectively, $N_{r} \times N_{\theta}=9250 \times 6400$ and $12.5 \mathrm{~m}$.

\section{Fast Rotation Case}

When the core of a progenitor star rotates rapidly with $\Omega_{\text {pre }} \sim \pi \operatorname{rad~s}^{-1}$, the rotation is the best agency to amplify the magnetic field. While winding of the magnetic field lines is one process of amplification, the so-called magnetorotational instability (MRI) is much more efficient (Balbus \& Hawley 1991, Akiyama et al. 2003). Since the fastestgrowing wavelength of the MRI is proportional to the magnetic field, a higher resolution is necessary for a weaker seed magnetic field. In the current case, the fastest-growing wavelength is a few $100 \mathrm{~m}$ near the surface of proto-neutron star. It is found that the magnetic field is exponentially amplified in some high resolution simulations. We infer this is due to the MRI by the fact that the growing time scale is comparable to one obtained by the linear analysis.

The impact of MRI is apparent by comparing low and high resolution simulations. As shown in Fig. 1, the high resolution simulations in which the MRI is resolved lead to a faster shock surface propagation compared to the low resolution simulations (and even a jet-like mass ejection in the high resolution model with the relatively strong magnetic field, $B_{\text {pre }}=2 \times 10^{11} \mathrm{G}$ ). We also found that the higher the resolution (equally the better the MRI resolved), the larger the explosion energy.

Interestingly, although the shock surface propagation is accelerated in the high resolution models shown here, magnetic pressure is not so large except for the vicinity of the rotation axis in the relatively strong magnetic field model (the upper right panel of Fig. 1), and it is even negligible in the most region in the weak magnetic field model (the lower right panel). This suggests that the explosion is not boosted by magnetic force.

We found that the boost of the explosion stems from an enhancement of neutrino heating due to indirect effects of the MRI. In the simulations in which the MRI is well resolved, the angular momentum is efficiently transferred outwards from the rotation axis as the growth of MRI proceeds. Then the region right behind the shock front gains more angular momentum and expands due to the extra centrifugal force. Since the neutrino heating surpasses the cooling more easily at a larger radius, the expansion leads to the enlargement of the gain region. Indeed, Fig. 2 shows that the gain region is larger in the higher resolution simulation. We also evaluated the ratio of the advection timescale, $\tau_{\mathrm{a}}$, during which matter traverses the gain region, to the heating timescale, $\tau_{\mathrm{h}}$, within which matter gains enough energy to overcome gravity. We found that the fraction increases more rapidly for a simulation of a better MRI resolution, indicating an enhancement of the neutrino heating.

\section{Slow Rotation Case}

When the rotation of the core is one order-of-magnitude slower than the former fast rotation case, the MRI is no longer an efficient amplification mechanism of the magnetic field, since its timescale, approximately equal to the rotation period, is now long compared 

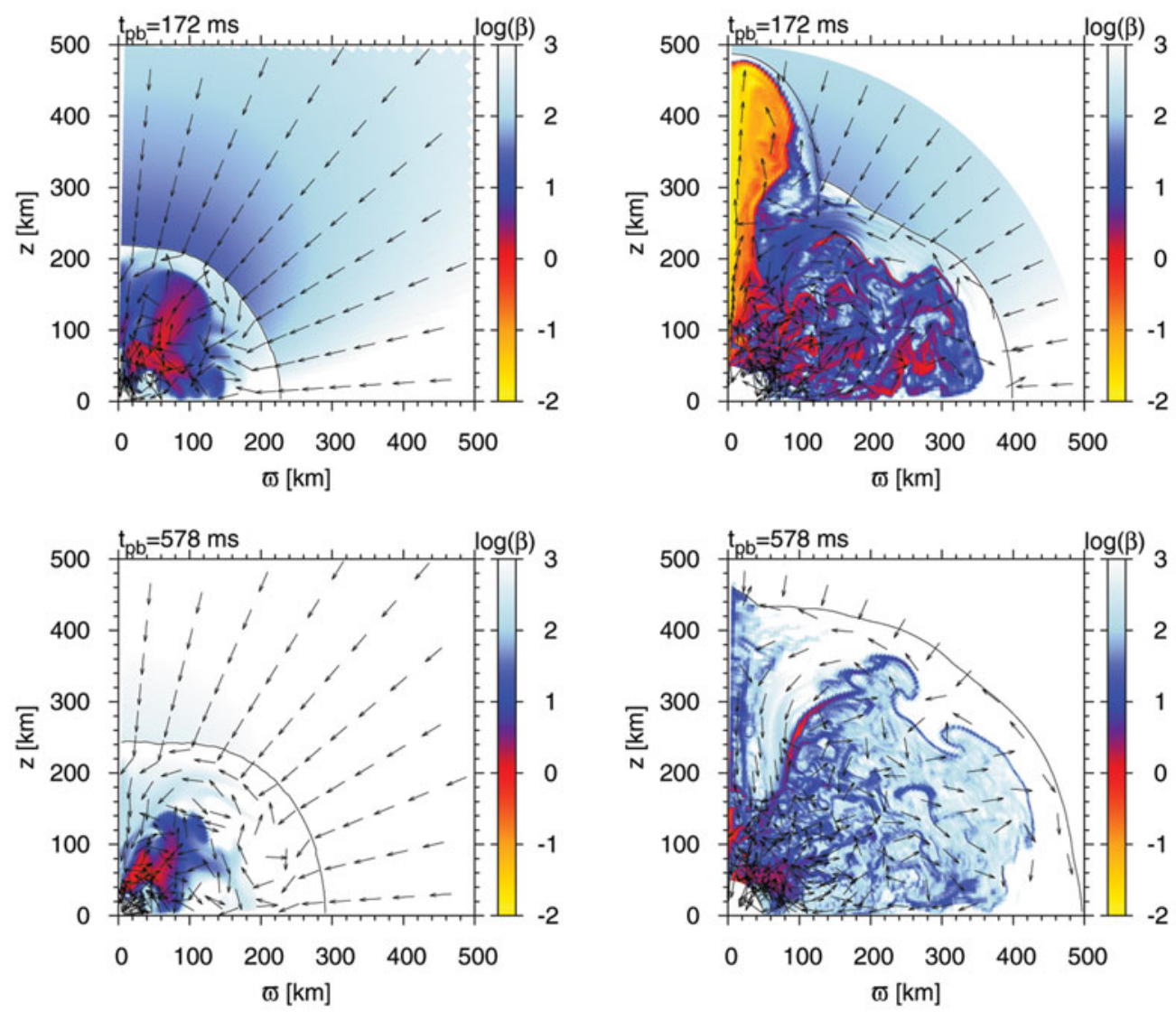

Figure 1. Color maps of the plasma beta with velocity vectors presented by arrows in the fast rotation case for models with $B$ pre $=2 \times 10^{11} \mathrm{G}$ at $t_{\mathrm{pb}}=172 \mathrm{~ms}$ (upper panels) and $B$ pre $=5 \times 10^{10} \mathrm{G}$ at $t_{\mathrm{pb}}=578 \mathrm{~ms}$ (lower panels). The left panels represent the results of low spatial resolution simulations with $720 \times 60$ grids while the right panels that of high spatial resolution simulations with $9250 \times 6400$ grids.
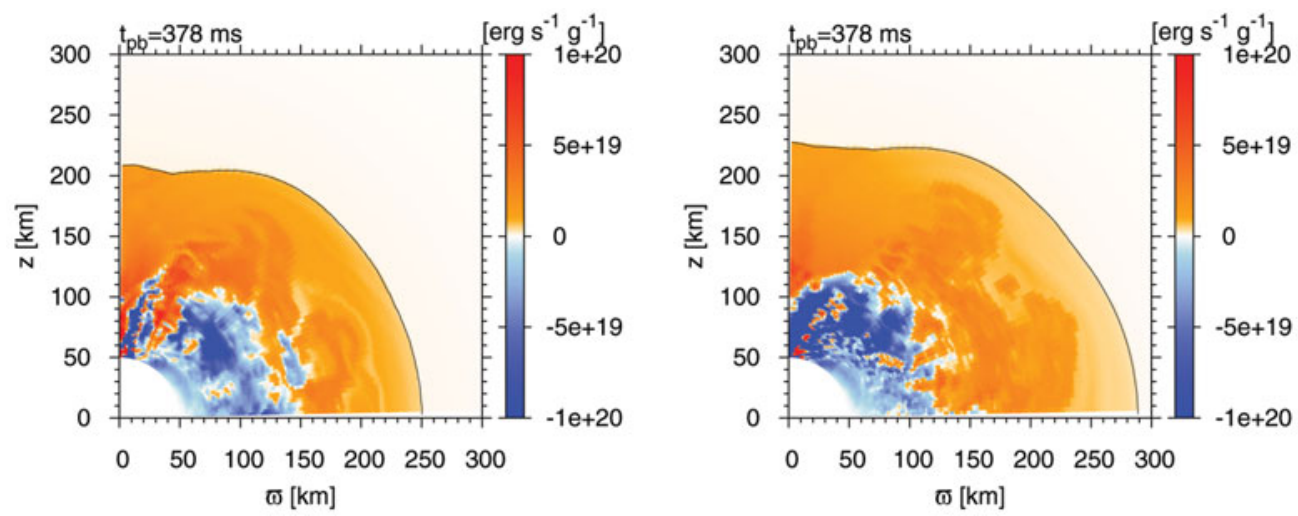

Figure 2. Color maps of the net heating rate per unit mass at $378 \mathrm{~ms}$ after bounce in the fast rotation case with $B$ pre $=5 \times 10^{10} \mathrm{G}$, for a low resolution simulation with $1160 \times 800$ grids (left) and for that of a high spatial resolution simulation with $9250 \times 1600$ grids (right). The volume of the (orange-colored) gain region is larger in the high resolution simulation. 

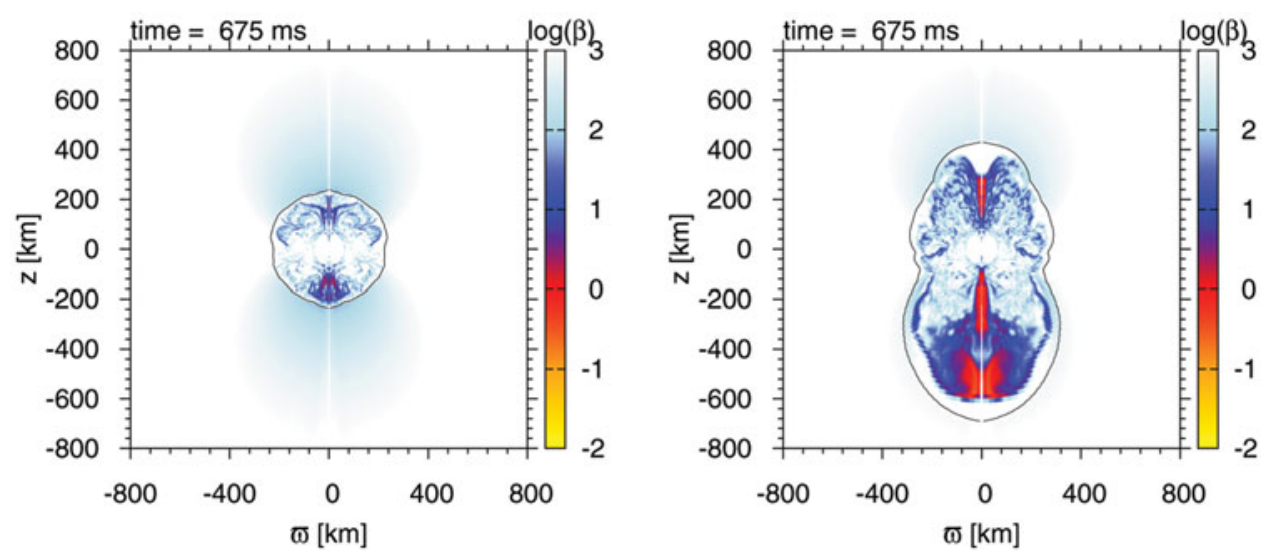

Figure 3. Color maps of the plasma beta in the slow rotation case with $B$ pre $=1 \times 10^{11} \mathrm{G}$ for a low resolution simulation with $1754 \times 1600$ grids (left), and for that of a high spatial resolution simulation with $3504 \times 3200$ grids (right). The explosion is only found in the latter case.

to the dynamical timescale around the surface of the proto-neutron star. Nevertheless, we found an exponential growth of magnetic field in this case too for simulations with high spatial resolutions. The amplification starts right after the shock generation and its timescale is comparable to the dynamical timescale, suggesting that the amplification is due to the buoyant convection caused by the shock propagation.

We found that the amplified magnetic field boosts the explosion again by increasing the neutrino heating, but in a different way from one shown in the previous section. Although the rotation is one order-of-magnitude slower than the previous case, it still works to substantially amplify the toroidal magnetic field in a very limited layer close to the rotation axis, where the rotational period reaches down to milliseconds. The amplified toroidal magnetic field weakly pushes the matter outwards, the shock surface expands accordingly, and by the same reason as before the neutrino heating is enhanced.

The right panel of Fig. 3 shows such an explosion. Comparison with the left panel prove the essential role of the magnetic field on the dynamics. Without the great amplification of the magnetic field, the matter is not lifted to a larger radius, the neutrino heating is inefficient, and the shock surface stalls at a small radius (the left panel).

\section{Conclusion}

We have seen the influences of magnetic field and rotation on core-collapse supernovae in the context of relatively weak magnetic fields. We found that they indirectly boost the explosion by enhancing the neutrino heating in either case where the progenitor rotates rapidly or slowly. In both cases, the enhancement of the neutrino heating occurs due to the expansion of the shock surface. In the former case, the expansion is caused by the angular momentum transfer accompanied by the MRI, while in the latter case, it is caused by the magnetic pressure amplified by convection and rotational winding of the magnetic field lines near the rotation axis. These results found by two-dimensional axisymmetric MHD simulations must be confirmed in three dimensional simulations, which may be feasible in the era of exascale computing.

\section{Acknowledgement}

Numerical computations in this work were performed on Cray XC30 at the Center for Computational Astrophysics, the National Astronomical Observatory of Japan. This 
work is supported by a Grant-in-Aid for Scientific Research from the Ministry of Education, Culture, Sports, Science and Technology, Japan (26800149).

\section{References}

Akiyama, S., Wheeler, J. C., Meier, D. L., \& Lichtenstadt, I. 2003, Astrophys J., 584, 954

Balbus, S. A. \& Hawley, J. F. 1991, Astrophys J., 376, 214

Janka, H.-T. 2001, Phys. Rep., 368, 527

Liebendörfer, M. 2005, Astrophys J., 633, 1042

Ramírez-Agudelo, O. H., Simón-Díaz, S., Sana, H., et al. 2013, Phys. Rep., 560, A29

Sawai, H. \& Yamada, S. 2014, Astrophys J. Lett., 784, L10

-. 2016, Astrophys J., 817, 153

Sawai, H., Yamada, S., Kotake, K., \& Suzuki, H. 2013, Astrophys J., 764, 10

Shen, H., Toki, H., Oyamatsu, K., \& Sumiyoshi, K. 1998a, Nuclear Physics A, 637, 435

-. 1998b, Progress of Theoretical Physics, 100, 1013

Woosley, S. E. \& Heger, A. 2006, Astrophys J., 637, 914

Woosley, S. E. \& Weaver, T. A. 1995, Astrophys J. Sup., 101, 181

Yamada, S. \& Sawai, H. 2004, Astrophys J., 608, 907 